\title{
Analysis of the TiO isotopologues in stellar optical spectra
}

\author{
Ya. V. Pavlenko ${ }^{1,2}$, Sergei N. Yurchenko ${ }^{3}$, Laura K. McKemmish ${ }^{4}$, and Jonathan Tennyson ${ }^{3}$ \\ ${ }^{1}$ Main Astronomical Observatory of NAS Ukraine, 27 Zabolotnoho, Kyiv 01137, Ukraine \\ e-mail: yp@mao.kiev.ua \\ ${ }^{2}$ Centre for Astrophysics Research, University of Hertfordshire, College Lane, Hatfield, AL10 9AB, UK \\ ${ }^{3}$ Department of Physics and Astronomy, University College London, London WC1E 6BT, UK \\ ${ }^{4}$ School of Chemistry, University of New South Wales, 2052 Sydney, Australia
}

Received 2 March 2020 / Accepted 4 August 2020

\begin{abstract}
Context. We used the new ExoMol TiO rovibronic line lists to identify and model TiO isotopologue features in spectra of $\mathrm{M}$ dwarfs. Aims. We investigate problems involving the computation of electronic bands for different isotopologues of TiO by modelling optical spectra of late-type stars. Based on this, we determine their Ti isotopic abundances and compare the TiO isotopologue spectra computed using line lists by different authors.

Methods. We fitted theoretical synthetic spectra to the observed stellar molecular bands of TiO. We modelled spectra of two M dwarfs, GJ 15A (M1V) and GJ 15B (M3 V), to determine Ti isotopic ratios in their atmospheres.

Results. We demonstrate the accuracy of the ExoMol Tото line list for different isotopologues of TiO and the possibility of determining accurate Ti isotope abundances in a number of spectral ranges. The 7580-7594 A spectral range seems particularly useful, with two atomic lines of $\mathrm{Fe} \mathrm{I}$ and molecular band heads of ${ }^{50} \mathrm{TiO},{ }^{49} \mathrm{Ti} \mathrm{O},{ }^{48} \mathrm{TiO}$, and ${ }^{47} \mathrm{TiO}$ clearly observable in our two $\mathrm{M}$-dwarf spectra. We determine non-solar Ti isotopic ratios of ${ }^{46} \mathrm{Ti},{ }^{47} \mathrm{Ti},{ }^{48} \mathrm{Ti},{ }^{49} \mathrm{Ti}$, and ${ }^{50} \mathrm{Ti}$ of $7.9,5.2,72.8,7.9$, and 6.2 for GJ $15 \mathrm{~A}$ and $7.4,4.2$, $76.6,5.8$, and 6.0 for GJ $15 \mathrm{~B}$ with an accuracy of \pm 0.2 . [Ti] $=0.040$ and 0.199 and within an accuracy of \pm 0.10 were also determined for GJ $15 \mathrm{~A}$ and GJ $15 \mathrm{~B}$, respectively.

Conclusions. We find that the ExoMol Tото TiO line list (a) describes the fine details in line position and intensity of the M-dwarf spectra better than other available $\mathrm{TiO}$ line lists, (b) correctly reproduces the positions and intensities of the TiO isotopologue band heads observed in M-dwarf spectra, and (c) can be used to determine Ti isotope abundances in atmospheres of M stars.
\end{abstract}

Key words. astrochemistry - stars: late-type - stars: abundances - molecular data - nuclear reactions, nucleosynthesis, abundances

\section{Introduction}

Naturally occurring titanium, the element of nuclear charge 22 , is composed of five stable isotopes, ${ }^{46} \mathrm{Ti},{ }^{47} \mathrm{Ti},{ }^{48} \mathrm{Ti},{ }^{49} \mathrm{Ti}$ and ${ }^{50} \mathrm{Ti}$. Thus far, nuclide abundances of these elements in the Solar System formed $4.56 \mathrm{Ga}$ ago have been measured with high accuracy. ${ }^{48} \mathrm{Ti}$ is the most abundant isotope $(73.72 \%$ natural abundance) with Solar System isotopic ratios of ${ }^{46} \mathrm{Ti},{ }^{47} \mathrm{Ti},{ }^{48} \mathrm{Ti},{ }^{49} \mathrm{Ti}$ and ${ }^{50} \mathrm{Ti}$ of 8.249, 7.372, 73.72, 5.409, and 5.185 (Lodders et al. 2009). Similar numbers are given by De Bievre \& Taylor (1993). Atoms of $\mathrm{Ti}$ isotopes in the low-temperature regime $(T<4000 \mathrm{~K})$ are bound in $\mathrm{TiO}$ molecules, and molecular bands of $\mathrm{TiO}$ are used to assess the low-mass stars and brown dwarfs of spectral class $\mathrm{M}$. Twenty-one radioisotopes of Ti have also been characterised, and the most stable is ${ }^{44} \mathrm{Ti}$ with a half-life of $60 \mathrm{yr} .{ }^{44} \mathrm{Ti}$ is observed in the supernova (SN) remnants, see Mochizuki et al. (1999). This isotope offers one of the most direct probes into nucleosynthesis environments in the interior of exploding stars, when the associated gamma-ray activities in the explosion remnant are detected and translated back into the isotopic abundances at the time of the explosion (Mochizuki \& Kumagai 2004; Mochizuki et al. 1999).

Details of the galaxy nucleosynthesis of $\mathrm{Ti}$ isotopes are described in many papers, see Hughes et al. (2008), for example. Sources of the stable Ti isotopes are known well, see Table 1 in Alexander \& Nittler (1999) and Woosley \& Weaver (1994):

$-{ }^{46} \mathrm{Ti}$ and ${ }^{47} \mathrm{Ti}$ are formed during explosive oxygen and silicon burning, respectively, in SN types II and Ia;
$-{ }^{48} \mathrm{Ti}$ is formed by explosive Si burning in type II SN;

$-{ }^{49} \mathrm{Ti}$ is formed by the explosive Si burning in type II SN;

$-{ }^{50} \mathrm{Ti}$ is formed in nuclear burning in type Ia SN.

Mochizuki et al. (1999) noted that long-lived isotopes with mean lifetimes of about $10^{6} \mathrm{yr}$ or longer will reflect a superposition of different $\mathrm{SNe}$ at different times, mixed with interstellar matter. Consequently, abundances in an individual object are formed by different branches of many processes at long timescales. Only short-lived isotopes will clearly trace individual events.

The classical model of Timmes et al. (1995) suggested that most stable $\mathrm{Ti}$ isotopes were under-produced with respect to solar values: relative to ${ }^{48} \mathrm{Ti}$ and the Solar System isotopic ratios, the predicted abundances of ${ }^{46} \mathrm{Ti}$ are a factor of two to high, those of ${ }^{47} \mathrm{Ti}$ are a factor of three too low, those of ${ }^{49} \mathrm{Ti}$ are an exact match, and those of ${ }^{50} \mathrm{Ti}$ are a factor of 2 too low. Isotopic ratios were predicted to decline with decreasing $[\mathrm{Fe} / \mathrm{H}]$ by a factor of 8 for ${ }^{46} \mathrm{Ti}$, a factor of 6 for ${ }^{47} \mathrm{Ti}$, a factor of 2 for ${ }^{49} \mathrm{Ti}$, and a factor of 30 for ${ }^{50} \mathrm{Ti}$ between $[\mathrm{Fe} / \mathrm{H}]=0$ and -1 . The degree of this underproduction depends on many theoretical assumptions, see Hughes et al. (2008).

Essentially, modelling the abundances of TiO isotopologues (Lambert et al. 1972; Wyckoff \& Wehinger 1972; Lambert \& Luck 1977; Clegg et al. 1979; Valenti et al. 1998) is the only way to extract $\mathrm{Ti}$ isotopic abundances from $\mathrm{M}$ stars. It is a very hard task to determine Ti isotopic ratios from the analysis of atomic lines because different isotopic lines have small isotopic 
Table 1. Parameters of stars taken from the literature.

\begin{tabular}{lllll}
\hline \hline$T_{\text {eff }}$ & $\log g$ & {$[\mathrm{Fe} / \mathrm{H}]$} & CompStar & Reference \\
\hline \multicolumn{5}{c}{ GJ 15A } \\
\hline 3606 & 4.93 & -0.27 & \multicolumn{3}{l}{ Passegger et al. (2018) } \\
3669 & & -0.29 & SUN & Gaidos et al. (2014) \\
3693 & & -0.26 & SUN & Gaidos \& Mann (2014) \\
3551 & & -0.28 & & Terrien et al. (2015) \\
3760 & & -0.23 & & Terrien et al. (2015) \\
3368 & & -0.26 & & Terrien et al. (2015) \\
3603 & & -0.30 & SUN & Mann et al. (2015) \\
3988 & & & & Gaia Collaboration (2018) \\
\hline & & & GJ 15B & \\
\hline 3283 & 5.11 & -0.19 & & Passegger et al. (2018) \\
3282 & & -0.17 & SUN & Gaidos et al. (2014) \\
3540 & 5.30 & -0.20 & SUN & Zboril \& Byrne (1998) \\
3254 & & -0.20 & SUN & Gaidos \& Mann (2014) \\
3218 & & -0.30 & SUN & Mann et al. (2015) \\
& & -0.08 & SUN & Newton et al. (2014) \\
3679 & 4.92 & -1.15 & SUN & Prugniel et al. (2011) \\
3630 & 4.71 & -0.88 & SUN & Koleva \& Vazdekis (2012) \\
3330 & 5.08 & -1.40 & SUN & Cenarro et al. (2007) \\
3636 & & & & Gaia Collaboration (2018) \\
\hline & & & &
\end{tabular}

shifts, see Kobayashi et al. (2019) and references therein. The broadening of spectral lines by macroturbulence in the stellar atmospheres as well as any notable rotation of stars almost prevents us from separating the atomic spectra of different isotopes (Tennyson 2019).

Accurate molecular line lists, that is, lists of energy levels and the intensity of spectral transitions, are crucial for correctly modelling a variety of astrophysical phenomena, including stellar photospheres and the atmospheres of extra-solar planets. Transition metal diatomic line lists are particularly difficult because of the large number of coupled low-lying electronic states (Tennyson et al. 2016b). TiO line lists are particularly important for studies of M-dwarfs (Pavlenko et al. 1995; Allard et al. 2000), and a number of them have been constructed (Schwenke 1998; Plez 1998; Ryabchikova \& Pakhomov 2015; McKemmish et al. 2019). The most recent of these is the TотO line list of McKemmish et al. (2019), which was computed as part of the ExoMol project (Tennyson \& Yurchenko 2012).

TiO line lists were first used to extract the titanium abundance in the 1970s, for instance, by Lambert et al. (1972), Wyckoff \& Wehinger (1972), Lambert \& Luck (1977) and Clegg et al. (1979). These studies were limited by the quality of the underlying line list, however, leading Valenti et al. (1998) to introduce isotope-dependent shifts to the line positions. These modifications should no longer be necessary with modern line list development techniques.

The majority of previous studies used the spectral range 7053.5-7055 $\AA$ to quantify the abundance of TiO isotopologues and thus the $\mathrm{Ti}$ abundance. However, the quality and availability of new and more accurate $\mathrm{TiO}$ molecular spectroscopic data and analysis techniques means that the suitability of this spectral region should be reconsidered.

We note that detailed comparisons with high-resolution Mdwarf spectra performed by McKemmish et al. (2019) showed that their Tото line list performed much better than the earlier line lists, particularly in matching line positions. These comparisons concentrated on the main isotopologue ${ }^{48} \mathrm{Ti}$. We assess here the utility of the Tото line list for modelling TiO isotopologue spectra in $\mathrm{M}$ dwarfs. A more detailed discussion of the Тото line list is given below. We also extended the comparison of the quality of $\mathrm{TiO}$ isotopologue line lists computed by different authors, see Sect. 2.1, by comparing fits of computed spectra to the observed spectra.

The nearest and brightest stars are among the best studied and hold a special place in popular imagination. The discovery of planets orbiting these stars tells us that the solar neighborhood is potentially rich with exoplanet systems; see Howard et al. (2014) for the complete list of known exoplanet-hosting stars within $7 \mathrm{pc}$. The great advantage of investigating these stars is that it is possible to undertake extensive campaigns to characterise them using a combination of high-resolution optical spectroscopy, near-infrared (NIR) spectroscopy, long-baseline optical/infrared interferometry, and high-cadence, broadband optical photometry. Even for the history of our own planetary system, the Ti isotopic studies are of great importance. Zhang et al. (2012) found that the ratio of ${ }^{50} \mathrm{Ti}$ to ${ }^{47} \mathrm{Ti}$ of the Moon is identical to that of the Earth within about four parts per million, which is only $1 / 150$ of the isotopic range documented in meteorites; see Table 2 in Zhang et al. (2012). This means that the Moonforming giant impactor, that is, hypothetical Theria, would be of the same isotopic composition as our Earth. Theory predicts that the Moon is constructed mostly (40-75\%) of the impactor materials. However, the observed identical titanium (and oxygen) isotopic compositions on Earth and the Moon are surprising in light of what we think we know about planet formation and formation of the Moon after a giant impact; see Taylor (2012). We expect growing interest of exoplanetary astrophysicists in isotopic studies of $\mathrm{Ti}$ and other elements.

We study the $\mathrm{TiO}$ isotopologues in spectra of the wide binary system GJ 15 A,B. GJ 15A (also known as Groombridge 34A, V* GX And, HD 1326A, HIP 1475, BD+43 44A, and Gaia DR2 385334230892516480) is a cool red dwarf of type M1. The other member of this binary star system, GJ 15B (also known as Groombridge 34B, V* GQ And, HD 1326B, BD+43 44B, and Gaia DR2 385334196532776576) is fainter and has a spectral type of M3.5 V (Reid et al. 1995). Lippincott (1972) measured a small astrometric segment of their orbit, giving an AB separation of $146 \mathrm{AU}$ and an orbital period of $2600 \mathrm{yr}$. Based on an imaging search for companions at $10 \mu \mathrm{m}$ with MERLIN at Palomar, van Buren et al. (1998) ruled out additional companions to A with projected separations of 9-36 AU with $T_{\text {eff }}>1800 \mathrm{~K}$ $\left(M>0.084 M_{\odot}\right)$. Gautier et al. (2007) found no IR excess for GJ $15 \mathrm{~A}$ at 24,70 , or $160 \mu \mathrm{m}$. Because GJ $15 \mathrm{~A}$ is a bright nearby star, many teams have provided studies of this component of the binary system; these are summarized in Table 1.

\section{Procedure}

We model optical spectra of $\mathrm{M}$ dwarfs. TiO features dominates here, but other molecules also provide notable features in different spectral ranges, see Pavlenko (2014) $)^{1}$. In addition to TiO, absorption by molecular bands of $\mathrm{VO}, \mathrm{CaH}, \mathrm{CrH}, \mathrm{MgH}$, and other hydrides from the Kurucz database (Kurucz 2011) were accounted for in our computations, as well as absorption by atomic lines taken from the VALD (Ryabchikova \& Pakhomov 2015).

1 Colour plots are available at www.mao.kiev.ua/staff/yp/ Results/M-stars/mb.htm 


\subsection{Titanium oxide line lists}

In the following, we analyse different line lists for monosubstituted isotopologues of $\mathrm{TiO}$ computed using the TотO line lists of McKemmish et al. (2019). The TOTO line lists were constructed for the main isotopologues of titanium oxide (TiO), namely ${ }^{46} \mathrm{TiO},{ }^{47} \mathrm{TiO},{ }^{48} \mathrm{Ti} \mathrm{O},{ }^{49} \mathrm{TiO}$, and ${ }^{50} \mathrm{TiO}$. Here and below we assume that oxygen is represented by its ${ }^{16} \mathrm{O}$ isotope as other oxygen isotopes are much less abundant in both the solar vicinity (De Bievre \& Taylor 1993; Lodders et al. 2009) and the majority of the known astrophysical objects.

The Тото line lists contain transitions with wavenumbers up to $30000 \mathrm{~cm}^{-1}$, that is, longwards of $330 \mathrm{~nm}$, and include all dipole-allowed transitions between 13 low-lying electronic states $\left(\mathrm{X}^{3} \Delta, \mathrm{a}^{1} \Delta, \mathrm{d}^{1} \Sigma^{+}, \mathrm{E}^{3} \Pi, \mathrm{A}^{3} \Phi, \mathrm{B}^{3} \Pi, \mathrm{C}^{3} \Delta, \mathrm{b}^{1} \Pi, \mathrm{c}^{1} \Phi, \mathrm{f}^{1} \Delta\right.$, and $\mathrm{e}^{1} \Sigma^{+}$). The Тото rovibronic line positrons were constructed using potential energy curves as simple Morse oscillators with constant diagonal and off-diagonal spin-orbit and other coupling terms fitted to match known empirical energy levels and ab initio curves where experimental data were unavailable. Accurate line intensities were generated using ab initio dipole moment curves. Final line lists were computed using the variational nuclearmotion program DUO (Yurchenko et al. 2016), where various couplings were explicitly included. The Tото line lists are appropriate for temperatures below $5000 \mathrm{~K}$ and contain about 60 million transitions for the main $\mathrm{TiO}$ isotopologue; higher temperature data are not required as $\mathrm{TiO}$ bands disappear in stellar spectra above about $4200 \mathrm{~K}$ due to dissociation of the titanium oxide molecule.

The variational procedure described above is known not to provide sufficiently accurate $\mathrm{TiO}$ spectra for high-resolution studies, see Hoeijmakers et al. (2015), for example. Therefore, McKemmish et al. (2019) used empirical energy levels they had obtained previously (McKemmish et al. 2017) using the so-called measured active rotation-vibration energy levels (MARVEL) procedure (Furtenbacher et al. 2007; Tóbiás et al. 2018) to improve the TOTO energy levels and thus the predicted transition frequencies. MARVEL energies are only available for ${ }^{48} \mathrm{Ti}^{16} \mathrm{O}$; the comparisons made by McKemmish et al. (2019) show that these corrected frequencies reproduce the majority of stellar features associated with $\mathrm{TiO}$ very well.

For the other isotopologues, McKemmish et al. (2019) adopted the method of Polyansky et al. (2017) and shifted the isotopologue energy levels by the observed (MARVEL) minus calculated value obtained for ${ }^{48} \mathrm{Ti} \mathrm{O}$. They made no allowance for any shifts associated with breakdown of the Born-Oppenheimer approximation. McKemmish et al. (2019) provided initial evidence for the success of this approach in the region 14145 $14175 \mathrm{~cm}^{-1}$ by comparing experimental $\mathrm{TiO}$ spectra to cross sections generated using the Тото line list and demonstrated that the weak peaks in the experimental spectra are isotopologue peaks. Our study provides a stringent test of how well this method works in practice.

The Tото TiO spectroscopic data is given in two datasets, a states and a transitions file (Tennyson et al. 2016a). The huge amount of data is not easy to use directly in astrophysical computations. Therefore we converted the Tото line data into the format of our synthetic spectra computation programs, see Table A.1. Furthermore, to reduce the number of lines that might effectively absorb radiation in the lower temperature regime we considered here, we selected only stronger lines using the cutoff parameter $\alpha>10-6$, here

$\alpha=g f * \exp \left(-E^{\prime \prime} / k T\right)$ where $f$ is the oscillator strength of line in absorption, $g$ is the statistical weight of the lower level, and $E^{\prime \prime}$ is the lower state energy, $T=3000 \mathrm{~K}$. After we computed the wavelength of the lines in vacuum, they were shifted to the air wavelength scale using the formulae of Ciddor (1996). In the following we use the air wavelength scale.

To estimate the quality of the different line lists, we compared fits to the observed spectra of GJ 15A and GJ 15B using line lists of TiO isotopologues computed by Schwenke (1998), labelled S98 below, Plez (1998), an updated version was taken from the web page ${ }^{2}$, labelled P12 below, and McKemmish et al. (2019), the Tото line list. The results of our comparison of synthetic spectra computed with different lists with observed spectra are given in Sects. 3.2.1 and 3.2.2.

\subsection{Observed spectra}

For the analysis, we used high-resolution spectra of both stars from the CARMENES spectral library of Reiners et al. (2018). The CARMENES spectra cover the wavelength range 520$1710 \mathrm{~nm}$ at a resolution of at least $R=80000$ or better. The spectra of GJ 15A and GJ 15B used in this work were observed with exposures in 61 and $198 \mathrm{~s}$ to obtain the comparable signalto-noise ratio $(\mathrm{S} / \mathrm{N})$ of 68 and 54 , respectively. We refer to Reiners et al. (2018) for more details. All theoretical spectra were shifted to account for the radial velocity of the appropriate star.

\subsection{Synthetic spectra and model atmospheres}

To generate synthetic spectra, we used the BT-Settl model atmosphere (Allard 2014). The procedure for computing synthetic spectra is described elsewhere; see Pavlenko (1997). We adopted the solar abundances of Anders \& Grevesse (1989). Line profiles were computed using Voigt profiles, and damping constants were taken from the line list databases or computed in the framework of the Unsöld approximation. Synthetic spectra were computed in wavelength steps of $0.025 \AA$, where we adopted the microturbulent velocity of $V_{\mathrm{t}}=1 \mathrm{~km} \mathrm{~s}^{-1}$. Some numerical experiments showed a rather marginal dependence of our results on $V_{\mathrm{t}}$. Our fitting procedure did not reveal any notable rotational velocities $v \sin i$, therefore the theoretical spectra were convolved with a pure Gaussian profile in order to model the instrumental broadening, see the next subsection. We fixed $T_{\text {eff }}=3800 \mathrm{~K}$ and $T_{\text {eff }}=3500 \mathrm{~K}$ for the $\mathrm{A}$ and $\mathrm{B}$ components, respectively. These values are not far from the Gaia 3869 and 3636 K (Gaia Collaboration 2018), respectively.

\subsection{Fits to the observed spectra}

The best fit to the observed spectra was achieved by the $\chi^{2}$ procedure described elsewhere (Pavlenko 2014). We give a few details here to aid understanding of our procedure. As part of the fit, the function

$S=\sum_{i=1}^{N} s_{i}^{2}$

is minimised, where $s_{i}=\left|F_{i}^{\mathrm{obs}}-F_{i}^{\mathrm{comp}}\right| ; F_{i}^{\mathrm{obs}}$ and $F_{i}^{\mathrm{comp}}$ are the observed and computed fluxes, respectively, and $N$ is the number of the wavelengths points used in the minimisation procedure. Three parameters were used in our minimisation procedure: the

2 https://nextcloud.lupm.in2p3.fr/s/r8pXijD39YLzw5T? path=\%2FTiOVALD 
Table 2. Spectral ranges of our interest.

\begin{tabular}{lcclc}
\hline \hline Sp. range & Wavelengths $(\AA)$, air & Wavenumber $\left(\mathrm{cm}^{-1}\right)$ & El. system & $\left(v^{\prime \prime}, v^{\prime}\right)$ \\
\hline$x_{1}$ & $7053.5-7055$ & $14173.2-14170.2$ & $\gamma\left(\mathrm{A}^{3} \Phi-\mathrm{X}^{3} \Delta\right)$ & $(0,0)$ \\
$x_{2}$ & $7580-7594$ & $13188.8-13164.5$ & $\gamma\left(\mathrm{A}^{3} \Phi-\mathrm{X}^{3} \Delta\right)$ & $(0,1)$ \\
$x_{3}$ & $8194-8204$ & $12200.5-12185.6$ & $\delta\left(\mathrm{b}^{1} \Pi-\mathrm{a}^{1} \Delta\right)$ & $(1,0)$ \\
$x_{4}$ & $8858-8862$ & $11286.0-11280.9$ & $\delta\left(\mathrm{b}^{1} \Pi-\mathrm{a}{ }^{1} \Delta\right)$ & $(0,0)$ \\
$x_{5}$ & $9720-9737$ & $10285.1-10267.1$ & $\delta\left(\mathrm{b}^{1} \Pi-\mathrm{a}^{1} \Delta\right)$ & $(0,1)$ \\
\hline
\end{tabular}

Doppler shift measured in $\mathrm{km} \mathrm{s}^{-1}$, the flux scale normalisation parameter, and the full width at half maximum (FWHM) used for the smoothing Gaussian. They were determined for every fitted spectral range. In our analysis we omitted some spectral ranges that contained artifacts provided by strong noise, telluric absorption, bad pixels, etc. The minimisation sum $S$ was computed on a $3 \mathrm{D}$ grid of radial velocity sets, flux normalisation factors, and FWHM parameters. Errors in the fit were evaluated as $\Delta S=\sum s_{i} / N$.

\section{Results}

\subsection{Search for the best spectral ranges}

Spectral features associated with isotopologues are difficult to see because they are faint and close to each other. The flux ratio method shows the strongest $\mathrm{TiO}$ absorption regions that should be analysed for the largest splitting. This should be complemented by observations of highest spectral resolution $(>100000)$ and highest S/N, see Valenti et al. (1998). It it worth noting here that usually, the macroturbulence in the atmospheres of late-type stars reduces the effective resolution to 70000 or less. Generally, the use of flux ratios could provide an alternative approach, but restricting ranges to the band heads allows the procedure to focus on measuring isotopic ratios. It is possible that the use of much wider spectral ranges may offer reasonable statistics on the accuracy of the individual wavelengths in the new line list with its better quality.

On the other hand, going to the shorter wavelengths may also enhance the chances of finding high-quality high-resolution spectra in observational archives (HIRES, HARPS, etc.), see Valenti et al. (1998) and others referenced in the introduction. However, this approach requires very high accuracy of the input line lists and even minimum blending with other molecular lines, even from the same molecule, is likely to cause problems. In practice, the $\mathrm{TiO}$ optical spectrum comprises absorption bands of different systems, and the responses of these band systems to changes in the temperature structure of the model atmosphere are different because of the different excitations of the lower level of each transition. Furthermore, using small spectral ranges is better than using a cross-correlation across a wide spectral range, and the $\mathrm{TiO}$ line list is less accurate at shorter wavelengths than longer wavelengths because we lack reliable spectroscopic data for the molecular levels with high excitation, see McKemmish et al. (2019) Conversely, we know from experience that an analysis of the well-characterised isotopologue band heads across comparatively short spectral ranges allows increasing the accuracy of the abundance determination by modelling infrared molecular bands of $\mathrm{CO}$ and $\mathrm{SiO}$, see Pavlenko et al. (2020) and references therein.

As a first step in our analysis, we therefore determined the spectral regions that are useful for an isotopic abundance determination of Ti. To this end, we computed two spectra of fluxes: the first spectrum, $F_{48}$, contains absorption of ${ }^{48} \mathrm{TiO}$ only, while the second spectrum $F_{\text {all }}$ contains all TiO isotopologues assuming the solar $\mathrm{Ti}$ isotopic ratios. Both spectra were smoothed by Gaussians with $R=60000$ and are shown in Fig. 1 together with their flux ratios: $F_{r}=F_{\text {all }} / F_{48}$. The flux ratios across band heads should show the highest sensitivity to the isotopologue abundances. Here we consider several spectral ranges with $\lambda>7000 \AA$. At shorter wavelengths, spectra of M stars become more complicated due to interference between different molecular bands while their flux also drops noticeably bluewards. We denote the spectral ranges of interests as $x_{1}, x_{2} \ldots x_{5}$ (see Table 2) and analyse the computed flux ratios in comparison with the observed X-shooter spectrum of GJ551 (Proxima Cen) as described by Pavlenko et al. (2017).

\subsubsection{Spectral range $x_{1}: 7053.5-7055 \AA$}

The main spectral feature of $x_{1}$ is formed by the $\mathrm{TiO} \gamma$-system, that is, $\mathrm{A}^{3} \Phi-\mathrm{X}^{3} \Delta,(0,0)$ band with its leading red-degraded band head at $7054 \AA$. This spectral window was used for modelling sunspot spectra by Lambert et al. (1972), Omicron Ceti by Wyckoff \& Wehinger (1972), Aldebaran by Lambert \& Luck (1977), spectra of late-type dwarfs and giants by Clegg et al. (1979), studies of M dwarfs by Valenti et al. (1998), and spectra of local M dwarfs by Chavez \& Lambert (2009). Unfortunately, the $\mathrm{TiO}$ band head in this region is formed by blends of unresolved components of $\mathrm{TiO}$ isotopologues, see the top right panel of Fig. 1, therefore we preferred to use wavelengths longer than the molecular head at $7054 \AA$. Furthermore, at the wavelength of the band head, there is a strong atomic line, clearly seen in the spectrum of Arcturus (Hinkle et al. 1995), which further complicates the analysis.

It is worth noting that this spectral range is also affected by absorption due to strong lines of the $\mathrm{TiO} \gamma^{\prime}(0,1)$ band, which is a part of the $\mathrm{B}^{3} \Pi-\mathrm{X}^{3} \Delta$ band system of the $(0,0)$ band head at $6192.5 \AA$. A mixture of lines of the $\gamma$ and $\gamma^{\prime}$ bands complicates the fine-structure analysis of the $x_{1}$ spectral range. Both bands are included in the TOTO line list.

\subsubsection{Spectral range $x_{2}: 7580-7594 \AA$}

The head of the red-degraded $(0,1)$ band of the $\gamma$-system $\left(\mathrm{A}^{3} \Phi-\right.$ $\left.\mathrm{X}^{3} \Delta\right)$ is located at $7589 \AA$. Here we have some contribution from lines in the tail of the $(0,0) \mathrm{TiO}$ band, which becomes stronger at lower $T_{\text {eff }}$.

Generally speaking, the fluxes in the optical spectra of latetype dwarfs increase towards the red, therefore this spectral range is preferable. On the other hand, the strong telluric $\mathrm{O}_{2} A$-band absorption is located approximately at $\lambda=7580 \AA$ (Rudolf et al. 2016). We note that the position of the molecular head in stellar 

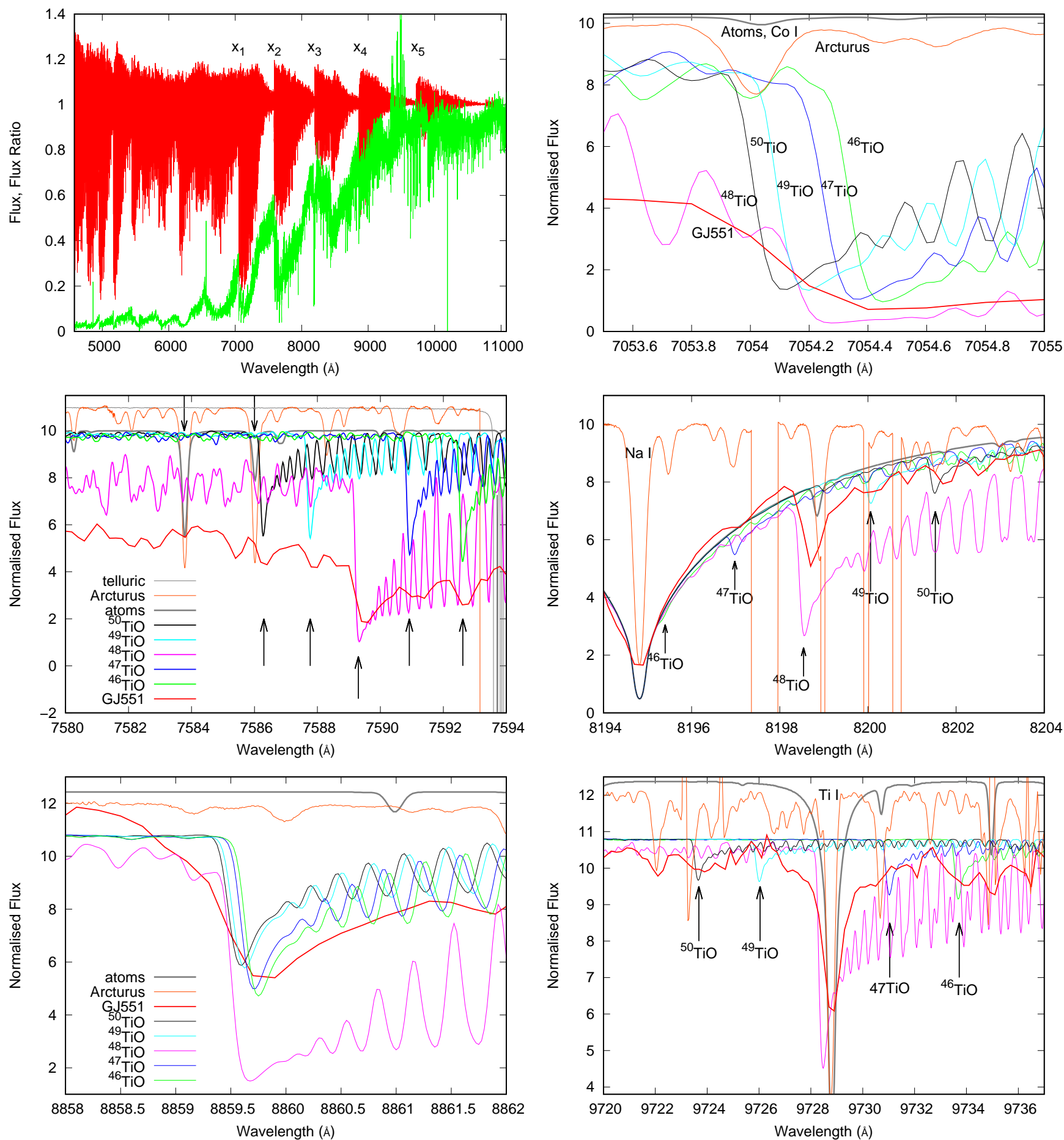

Fig. 1. Top left: observed X-shooter spectrum of GJ551 (green line) (Pavlenko et al. 2017) and flux ratio $F_{48} / F_{\text {all }}$ (red line); the green line shows the observed X-shooter fluxes in the Proxima spectrum. $x_{1}, \ldots x_{5}$ mark our spectral ranges of interest. Top right: band heads of TiO isotopologues computed for a 2900/4.5/0.0 model atmosphere. All observed spectra are shifted to the zero radial velocity frame. Middle panels: theoretical spectra of bands of TiO isotopologues computed across the $x_{2}$ it (right panel) and $x_{3}$ (left panel) spectral ranges. Upward arrows show the positions of head bands of the TiO isotopologues. Downward arrows label two Fe I lines seen in absorption. Bottom panels: theoretical spectra of bands of $\mathrm{TiO}$ isotopologues computed across $x_{4}$ (right panel) and $x_{5}$ (left panel) spectral ranges. The positions of the molecular band heads are shown by arrows. In all panels the observed spectra of Acturus and Proxima Cen (GJ551) are shown by thin and thick red lines, respectively, to demonstrate the locations of notable atomic and molecular features in the observed spectra.

spectra reduced to the local coordinate frame varies depending on the relative radial velocity of the star. In the X-shooter spectrum of Proxima Cen obtained with long exposures, the $\mathrm{O}_{2}$ molecular band is found at even shorter wavelengths, that is, beyond $7580 \AA$. There are two atomic lines of Fe I in the $x_{2}$ spectral range that provide the means to determine accurate Doppler shifts that are important for the proper identification of the weak $\mathrm{TiO}$ isotopologue features. 
As shown in the left middle panel of Fig. 1, features created by ${ }^{50} \mathrm{TiO},{ }^{49} \mathrm{TiO}$, and ${ }^{48} \mathrm{TiO}$ are clearly seen in the computed HIRES spectra as well as in the X-shooter spectrum of intermediate resolution of Proxima. The heads of the isotopologue bands ${ }^{46} \mathrm{TiO}$ and ${ }^{47} \mathrm{TiO}$ can be seen only in the background of stronger lines belonging to the tail of ${ }^{48} \mathrm{TiO}$ in the HIRES spectra.

An additional advantage of the $x_{2}$ spectral region is the presence of two atomic Fe I lines at 7583.8 and $7586.0 \AA$, see the downward arrows in the middle left panel of Fig. 1. These lines are clearly seen in the Arcturus spectrum and can be used for an accurate wavelength reduction in cooler stars as well.

\subsubsection{Spectral range $x_{3}: 8194-8204 \AA$}

The $x_{3}$ spectral range contains the $(1,0)$ band head of the $\delta$ $\left(b^{1} \Pi-a^{1} \Delta\right.$ ) system of TiO. The main absorption feature here is formed by the reddest line of the subordinate $\mathrm{Na} I$ triplet at 8183.255, 8194.790, and $8194.823 \AA$ (8200 A triplet), see the right middle panel of Fig. 1. Interestingly, the molecular bands occur in reverse order in comparison to the $\gamma$ band system; here the heads of the isotopologue bands occur in ascending order, from ${ }^{47} \mathrm{TiO}$ to ${ }^{50} \mathrm{TiO}$.

Unfortunately, this structure of the $x_{3}$ spectral range complicates the detailed analysis of the weak molecular features created by the bands of $\mathrm{TiO}$ isotopologues. Subordinate $\mathrm{Na}$ I lines of the $8200 \AA$ triplet are well-known indicators of the gravity in late-type dwarfs, but they depend on the effective temperature as well, see Schlieder et al. (2012). Likely, the $x_{3}$ wavelength range can be used for Ti isotopic analysis in spectra of lower gravity stars, in which Na I lines at $8200 \AA$ should have weaker wings.

\subsubsection{Spectral range $x_{4}: 8858-8862 \AA$}

The strong head of the $(0,0)$ band of the $\delta$ system, that is, $\mathrm{b}^{1} \Pi$ $a^{1} \Delta$, is a notable observational feature in the $x_{4}$ range. Unfortunately, the wavelength shifts between heads of $\mathrm{TiO}$ isotopologue bands are small so that even in the HIRES spectra we can see only one band head formed by several TiO isotopologues bands. The tail of the composite molecular band provides some opportunities for detecting isotopologue lines that are notably shifted with respect to the ${ }^{48} \mathrm{TiO}$ lines; these can be seen in the 88818862 Å range where strong atomic or telluric lines are absent. On the other hand, these wavelengths are severely polluted by $(0,0)$ $\mathrm{FeH}$ and $\mathrm{CrH}$ molecular lines of the $\mathrm{FeH} \mathrm{F}{ }^{4} \Delta_{i}-\mathrm{X}^{4} \Delta_{i}$ and $\mathrm{CrH}$ A ${ }^{6} \Sigma^{+}-\mathrm{X}^{6} \Sigma^{+}$systems with band heads at 8694 and $8520 \AA$, respectively, see Pavlenko (2014).

\subsubsection{Spectral range $x_{5}: 9720-9737 \AA$}

The $x_{5}$ spectral range contains the $(0,1)$ band head of the $\delta\left(\mathrm{b}^{1} \Pi-\mathrm{a}^{1} \Delta\right)$ system of TiO. The bottom right panel of Fig. 1 illustrates at least two problems of this wavelength range: (a) the spectrum is severely polluted by telluric absorption, and (b) the strong Ti I atomic line at $9728.32 \AA$ seen in the spectrum of Arcturus and Proxima forms blend with a molecular band head of ${ }^{48} \mathrm{TiO}$. On the other hand, the $\mathrm{TiO}$ isotopologue band heads are well spaced in wavelength. Again, molecular bands of higher mass isotopologues are shifted blue-ward, away from the strong ${ }^{48} \mathrm{TiO}$ band head. The $x_{5}$ spectral range can be used to determine ${ }^{50} \mathrm{TiO}$ and ${ }^{49} \mathrm{Ti} \mathrm{O}$ abundances, but only in the of absence of telluric absorption at the wavelengths of their band heads. The ${ }^{47} \mathrm{TiO}$ and ${ }^{46} \mathrm{Ti} \mathrm{O}$ band heads can be observed in the background of the stronger tail of the ${ }^{48} \mathrm{TiO}$ band. Again, the strong telluric absorption makes it difficult to perform a fine-structure analysis of the weak molecular features across this spectral range.

\subsection{Determination of Ti isotope abundances in the atmospheres of GJ $15 A$ and GJ 15B}

After selecting the best spectral ranges for the determination of Ti isotope abundances, we performed fits of our synthetic spectra to observed spectra of GJ 15A and GJ 15B in selected spectral ranges to demonstrate that they can be used to determine the abundances of Ti isotopes in their atmospheres. As before, we assumed that all oxygen atoms exist in the form ${ }^{16} \mathrm{O}$.

\subsubsection{Spectral range $x_{1}$}

As shown in Sect. 3.1.2, the heads of the $(0,1)$ band of the $\gamma$-system of TiO isotopologues form an unresolved blend at $7054 \AA$, even in HIRES spectra obtained with $R \sim 70000$. Some authors used spectral regions beyond $7054 \AA$ to determine the TiO isotope abundance. Spectral ranges $\sim 7071.5-7075 \AA$ and 7079-7082.5 were used by Lambert \& Luck (1977) and Clegg et al. (1979) to analyse spectra of M stars. A detailed identification of positions of $\mathrm{TiO}$ isotopologues is given in their papers. However, we see from their identifications that nearly all wavelengths receive a contribution from several $\mathrm{TiO}$ isotopologues. To illustrate this point, the two upper panels of Fig. 2 show spectra of all $\mathrm{TiO}$ isotopologues computed separately. We show here fluxes that were used to create the upper left panel of Fig. 1; at larger scales, these fluxes are shown for the above spectral ranges in the wavelength frame reduced to $V_{r}=0$. The strongest features are marked by atomic numbers $z$ of Ti atoms formed by corresponding $\mathrm{TiO}$ molecules.

The lower panels of Fig. 2 show fits to spectra of GJ 15A and $\mathrm{B}$ for the cases of the solar isotopic ratios of $\mathrm{Ti}$, that is, ${ }^{46} \mathrm{Ti}$, ${ }^{47} \mathrm{Ti},{ }^{48} \mathrm{Ti},{ }^{49} \mathrm{Ti}$, and ${ }^{50} \mathrm{Ti}$ of 8.3, 7.4, 73.7, 5.4, and 5.2 (De Bievre \& Taylor 1993; Lodders et al. 2009), and a modified Ti isotopic ratio, ${ }^{46} \mathrm{Ti},{ }^{47} \mathrm{Ti},{ }^{48} \mathrm{Ti},{ }^{49} \mathrm{Ti}$, and ${ }^{50} \mathrm{Ti}$ of $10.4,9.3,67.0,6.8$, and 6.5 , in the latter case, the abundances of ${ }^{48} \mathrm{Ti}$ were reduced by $10 \%$, and the abundances of the other Ti isotopes were increased by $25 \%$. For simplicity we label these modified TiO isotopic abundances as the M25 ratios. The comparison of two spectra illustrates the sensitivity of the selected spectral features to changes of Ti isotopic ratio.

We note that all isotopologue features can be clearly identified in the observed spectra. However, we see only a rather marginal response in these features to the change in Ti isotopic ratios. In all cases we see blends of a few lines of different isotopes, and the dominant contribution of ${ }^{48} \mathrm{TiO}$. The lines of ${ }^{48} \mathrm{TiO}$ are saturated, which means that they show a rather weak response to isotopic abundance changes. A qualitative byeye estimate of the comparison of the fine details between the observed and computed spectra seems to suggest a better fit for the case of solar Ti isotope ratios. These fits adopted [Ti] = $A_{\odot}(\mathrm{Ti})-A_{*}(\mathrm{Ti})=-0.1$, where $A_{\odot}(\mathrm{Ti}), A_{*}(\mathrm{Ti})$ are abundances of Ti in the atmospheres of the Sun and star, for GJ 15A and -0.2 for GJ 15B.

Fits to the $x_{1}$ spectral range for spectra of GJ $15 \mathrm{~A}$ and GJ 15B using three line lists for $\mathrm{TiO}$ isotopologues are presented: S98 from Schwenke (1998), P12 updated from Plez (1998), and Тото of the Exomol group (McKemmish et al. 2019). Plots with the fits are given in Fig. A.1, which shows fits to the observed spectra in the selected regions, as also illustrated in Fig. 2. We 

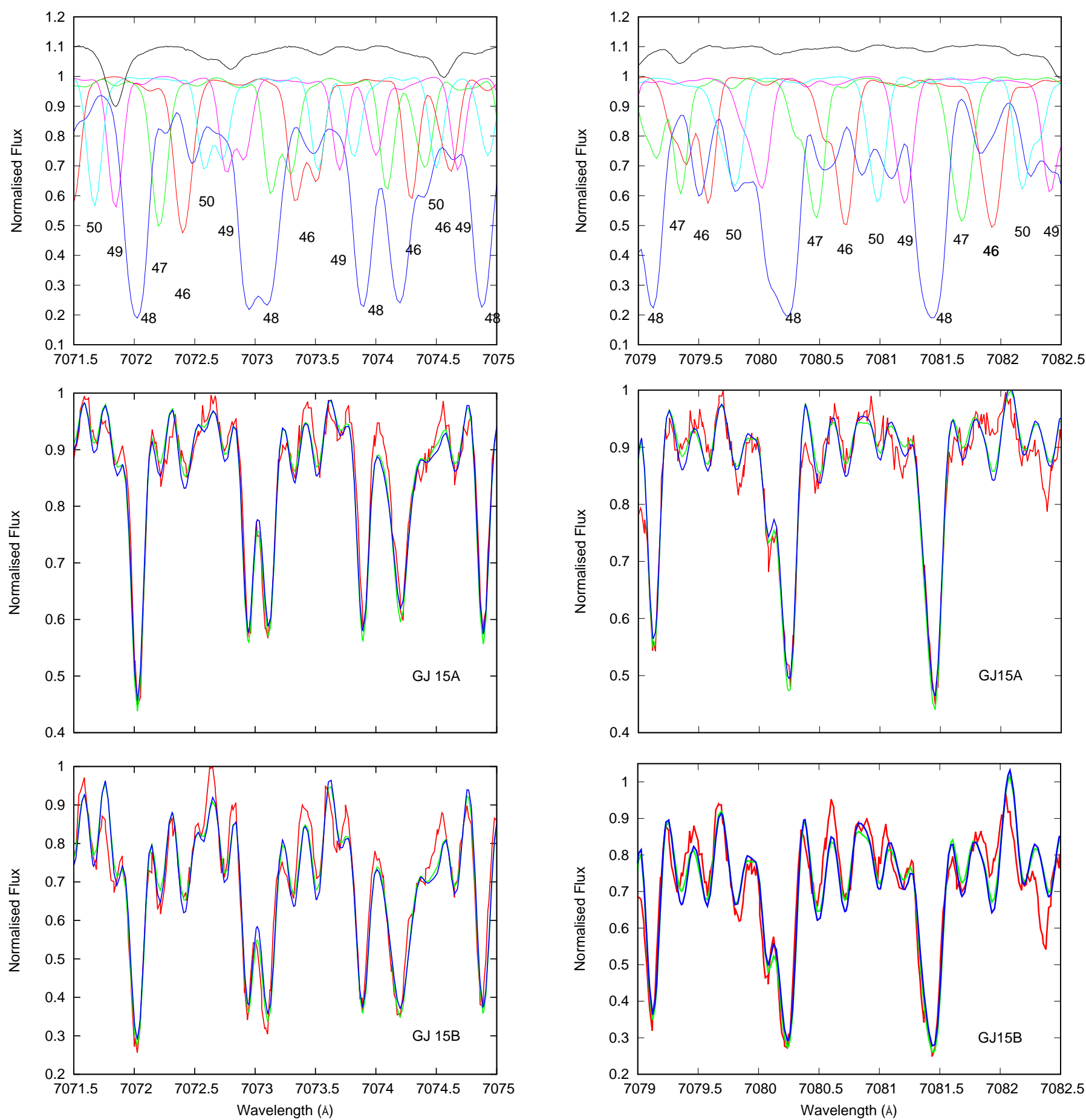

Fig. 2. Upper panels: features created by the TiO isotopologues in the spectral ranges used by Lambert \& Luck (1977) and Clegg et al. (1979) to determine the Ti isotopic abundance in M-stars. Theoretical spectra of TiO isotopologues were computed for 3500/5.0/0.0 model atmosphere and solar Ti isotopic ratios. Synthetic spectra are convoluted with $R=70000$. The strongest features are marked by atomic numbers of the Ti isotope in the associated TiO molecule. Black line shows the Arcturus spectrum (Hinkle et al. 1995). Middle panels: fits of theoretical spectra computed for 3800/5.0 model atmospheres to the observed spectra of GJ 15A. Bottom panels: fits of theoretical spectra computed for 3500/5.0 model atmospheres to the observed spectra of GJ 15B. Observed spectra are denoted by red lines, spectra computed for the solar isotopic abundance are shown by green lines, and spectra computed for M25 isotopic ratios by blue lines.

performed fits to the head of the $\gamma$ system at $7054 \AA$. The computed $\chi^{2}$ minimisation sums are given in Table 3 . In all cases, Тото provides the best fit. We list the conclusions below that we also made from the comparisons.

a) The positions of the strong absorption feature formed by absorption of photons in the ${ }^{48} \mathrm{Ti}$ transitions agree well, but the intensities disagree in some cases. b) We see more pronounced differences in the positions and intensities for lines belonging to other less abundant $\mathrm{TiO}$ isotopologues.

c) The formally computed $S$ parameters that we obtained using different line lists differ from each other, see Table 3.

We note that this rather narrow and phenomenological comparison of the results obtained with different line lists cannot 

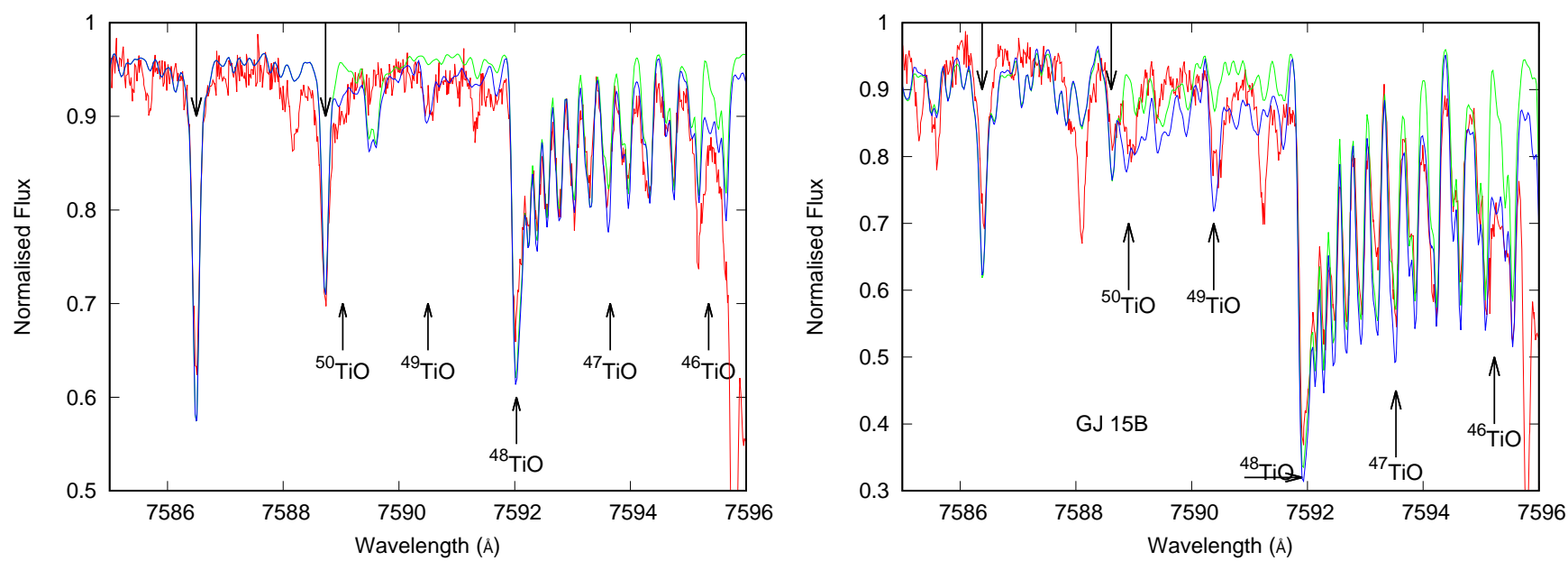

Fig. 3. Fits of our synthetic spectra to the observed GJ 15A (left panel) and GJ 15B (right panel) spectra with $\mathrm{Ti}$ isotopic ratios ${ }^{50} \mathrm{Ti}$, ${ }^{49} \mathrm{Ti},{ }^{48} \mathrm{Ti}$, ${ }^{47} \mathrm{Ti}$, and ${ }^{46} \mathrm{Ti}$ of $6.2,7.9,72.8,5.2$, and 7.9 and $6.0,5.8,76.6,4.2$, and 7.4 , respectively, shown by blue lines. Observed spectra are shown by red lines, and fluxes computed for ${ }^{48} \mathrm{Ti} \mathrm{O}$ alone are shown by green lines. Downward arrows mark $\mathrm{Fe} \mathrm{I}$ lines.

Table 3. Parameter $S$ computed in the fitting ranges given as wavelengths in $\AA$.

\begin{tabular}{lccl}
\hline \hline & $7053-7060$ & $7071-7084$ & $7580-7594$ \\
\hline P12 & $0.73 \pm 0.03(\mathrm{~A})$ & $2.75 \pm 0.04$ & $0.75 \pm 0.02$ \\
& $2.11 \pm 0.05(\mathrm{~B})$ & $7.60 \pm 0.08$ & $3.50 \pm 0.04$ \\
S98 & $0.66 \pm 0.03(\mathrm{~A})$ & $2.45 \pm 0.04$ & $0.76 \pm 0.02$ \\
& $1.64 \pm 0.04(\mathrm{~B})$ & $3.64 \pm 0.05$ & $3.40 \pm 0.04$ \\
TOTO & $0.47 \pm 0.02(\mathrm{~A})$ & $1.10 \pm 0.03$ & $0.73 \pm 0.02$ \\
& $1.28 \pm 0.04(\mathrm{~B})$ & $2.32 \pm 0.04$ & $3.19 \pm 0.04$ \\
\hline
\end{tabular}

provide a final conclusion about which is the best list. However, our conclusions are valid for three spectral ranges, which have been used by different authors for Ti isotopic abundance analysis.

However, when we combine our findings with the more sophisticated, deeper, and independent analysis made by McKemmish et al. (2019) and Bernath (2020), we find general agreement that Тото is the best list. Even the by-eye inspection confirms that the TOTO line list is better at reproducing the observed features in terms of both line intensities and line positions, see Fig. 3.

\subsubsection{Spectral range $x_{2}$}

The spectral range $x_{2}$ is favourably different from $x_{1}$. Heads of the TiO bands are well spaced across the wavelengths, which improves their identification and analysis. This is more relevant for the band heads of ${ }^{50} \mathrm{TiO}$ and ${ }^{49} \mathrm{Ti} \mathrm{O}$, which are located before the head of the strong ${ }^{48} \mathrm{TiO}$ band. Despite the strong blending between the ${ }^{50} \mathrm{TiO}$ band head and an Fe I line, the spectral detail is clearly seen in the observed spectrum, which is relevant to stars with low $v \sin i$.

Unfortunately, in the spectra of the two stars, the ${ }^{46} \mathrm{Ti} \mathrm{O}$ band head coincides with strong telluric feature. Still, molecular band heads of other $\mathrm{TiO}$ isotopologues are seen, therefore we were able to determine all ratios. ${ }^{46} \mathrm{Ti}$ was determined as $100-\left({ }^{47} \mathrm{Ti}+\right.$ $\left.{ }^{48} \mathrm{Ti}+{ }^{49} \mathrm{Ti}+{ }^{50} \mathrm{Ti}\right)$. Moreover, we included [Ti] in the minimisation process because the isotopic ratios depend on the abundance of the element, see Fig. 2 in Pavlenko et al. (2020) for the case of
Table 4. Isotopologue abundances and $\mathrm{Ti}$ abundance in the atmospheres of GJ $15 \mathrm{~A}$ and GJ $15 \mathrm{~B}$.

\begin{tabular}{cccc}
\hline \hline Isotopologue & The Sun & GJ 15A & GJ 15B \\
\hline${ }^{46} \mathrm{Ti}$ & 8.3 & $7.9 \pm 0.01$ & $7.4 \pm 0.3$ \\
${ }^{47} \mathrm{Ti}$ & 7.4 & $5.2 \pm 0.01$ & $4.2 \pm 0.4$ \\
${ }^{48} \mathrm{Ti}$ & 73.7 & $72.8 \pm 0.01$ & $76.6 \pm 0.2$ \\
${ }^{49} \mathrm{Ti}$ & 5.4 & $7.9 \pm 0.01$ & $5.8 \pm 0.3$ \\
${ }^{50} \mathrm{Ti}$ & 5.2 & $6.2 \pm 0.01$ & $6.0 \pm 0.0$ \\
{$[\mathrm{Ti}]$} & 0.0 & $0.040 \pm 0.001$ & $0.199 \pm 0.003$
\end{tabular}

the carbon isotopic ratio determination. Strong and weak features show different responses to abundance variations.

We performed the isotopologue abundance analysis using the determination of $\min S\left({ }^{47} \mathrm{Ti},{ }^{48} \mathrm{Ti},{ }^{49} \mathrm{Ti},{ }^{50} \mathrm{Ti}\right.$, and [Ti]) for the set of synthetic spectra computed for the grid of input parameters given below:

$-{ }^{47} \mathrm{Ti}$ varies in the range [3.0-10.0] with a step of 1.0;

$-{ }^{48} \mathrm{Ti}$ varies in the range [71.5-77.5] with a step of 0.5 ;

$-{ }^{49} \mathrm{Ti}$ varies ithe range [3.0-10.0] with a step of 1.0 ;

$-{ }^{50} \mathrm{Ti}$ varies in the range [3.0-10.0] with a step of 1.0 ;

$-[\mathrm{Ti}]$ varies in the range $[0-0.1]$ and $[0.1-0.2]$ with steps of 0.02 for GJ 15A and GJ 15B, respectively.

These parameter ranges were determined in a set of numerical experiments.

Our 5D minimisation procedure provides a set of minimisation sums, $S$, followed by formally computed errors, $\Delta S$. We obtained $S_{\min }=1.030 \pm 0.021$ for GJ $15 \mathrm{~A}$, and then averaged solutions falling in the window $1.030<S<1.052$. For GJ 15B we averaged solutions in the range $3.332<S<3.375$. Because the level of noise in the GJ 15B spectrum is higher, our solution is less reliable for this cooler star. Table 4 shows relative abundances of TiO isotopologues determined from the $\chi^{2}$ fits to the observed spectra of GJ 15A and GJ 15B.

The green lines in Fig. 3 show spectra computed without ${ }^{46} \mathrm{Ti} \mathrm{O},{ }^{47} \mathrm{Ti} \mathrm{O},{ }^{49} \mathrm{Ti} \mathrm{O}$, and ${ }^{50} \mathrm{Ti} \mathrm{O}$. The comparison with the full sample of $\mathrm{TiO}$ isotopologues shows the contribution of the less abundant species to the total spectrum.

Generally speaking, in the ideal case of isolated stars of similar masses that form a wide binary system, we do not expect 
large differences in their abundances. As a result, any differences in isotopic ratios may be caused by other reasons, which might tell us something about our procedure or about the physical state of the stars. Here, we find good agreement in the fits to the ${ }^{49} \mathrm{Ti} \mathrm{O}$ and ${ }^{47} \mathrm{Ti} \mathrm{O}$ features, but we also see some disagreement for ${ }^{50} \mathrm{Ti} \mathrm{O}$ in the shape and intensity between the computed molecular bands and the observations.

To compare the fits of synthetic spectra in the $x_{2}$ spectral using the different line lists S98, P12, and Tото we computed the parameter. In these computations we adopted solar isotopic ratios for the $\mathrm{Ti}$ isotopes. The fits to the observed spectra are shown in Fig. A.2; the computed values of $S$ for all of them are given in Table 3.

We note that the two Fe I lines seen in this spectral range can be fitted with $[\mathrm{Fe}]=-0.4$ for both stars, which confirms the deficit of iron found previously, see Table 1. Our minimisation procedure allows us to determine $[\mathrm{Ti}]=0.040 \pm 0.001$ and $0.199 \pm 0.003$ for GJ $15 \mathrm{~A}$ and GJ $15 \mathrm{~B}$, respectively.

\subsubsection{Spectral ranges $x_{3}-x_{5}$}

Unfortunately, $\mathrm{TiO}$ is weak in the $x_{3}-x_{5}$ spectral ranges for the observed and computed spectra of GJ 15A and GJ 15B. The $x_{3}$ spectral range is dominated by an $\mathrm{Na} \mathrm{I}$ line; here we see only a weak ${ }^{48} \mathrm{TiO}$ band head. This spectral range may be more useful for giant spectra, where the Na I $8200 \AA$ subordinate triplet is much weaker. The isotopologue bands are here in ascending order, that is, from ${ }^{46} \mathrm{Ti} \mathrm{O}$ to ${ }^{50} \mathrm{TiO}$, which is the opposite order to the $x_{2}$ spectral range.

As we noted above, the molecular bands of the TiO isotopologues in the $x_{4}$ spectral range form a single feature in the observed and computed spectra, as in $x_{1}$ spectral range. We cannot distinguish contributions from $\mathrm{TiO}$ isotopologues in the spectra of GJ 15A and GJ 15B because TiO appears much weaker here and there is strong pollution by telluric lines.

In the $x_{5}$ spectral range we observe a descending order of the isotopologue bands, similar to $x_{2}$. However, the $\mathrm{TiO}$ absorption is too weak here to form any notable features that might be used in the analysis.

\section{Discussion}

Although molecular spectroscopy of $\mathrm{TiO}$ provides the most accurate method of determining the titanium isotope abundance in cool stars, the analysis of $\mathrm{TiO}$ spectroscopy is very challenging. As a heavy molecular system, the $\mathrm{TiO}$ spectra is complex, with spectral features that formed by blends of many absorption lines, often from different isotopologues. To obtain high-accuracy measurements, it is vital to select the best spectral region for the high-resolution analysis.

The main aims of this paper are (a) to test the new Tото $\mathrm{TiO}$ line list to determine the $\mathrm{TiO}$ isotopologue abundances in the observed spectra of cool M dwarfs, (b) to determine the most appropriate spectral ranges in which the $\mathrm{Ti}$ isotope abundances in their atmospheres can be determine, and (c) to retrieve the Ti isotopic ratios for GJ $15 \mathrm{~A}$ and GJ $15 \mathrm{~B}$ to test the performance of this method.

\subsection{Line list accuracy}

Previous comparisons of $\mathrm{TiO}$ line lists (e.g. by McKemmish et al. 2019; Bernath 2020; Piette et al. 2020) have concluded that the Тото line list is generally superior to previously assembled line lists. However, these comparisons did not explicitly consider the quality of the predicted isotopologue spectra, that is, how accurately each line list reproduces the astronomically observed isotopologue frequency shifts and therefore allows the abundance of different titanium isotopes to be accurately measured astronomically. We here modelled several spectral regions that can be used for a Ti isotope abundance analysis in cool stars, and we compared our model isotopologue spectra that were computed using the Тото line list with other available line lists. The detailed comparisons with high-resolution M-dwarf spectra with different line lists showed that the Tото line list performs much better than the earlier line lists, particularly in matching line positions, see Fig. A.1.

\subsection{Best spectral ranges for isotope determination}

In the past, most researchers have concentrated on analysing the fine details of absorption spectra formed in the redward degrading $(0,0)$ band of the TiO $\gamma$ band system. However, observable features here are formed by blends of many lines belonging to different isotopologues. In our case, synthetic spectra computed for the solar Ti isotopic ratios in our $x_{1}$ spectral range can be fitted to the observed spectra of GJ 15A and GJ 15B; but we found here a rather weak dependence on the reasonable changes $(25 \%)$ of the $\mathrm{TiO}$ isotopologue abundances.

We investigated a few possible spectral ranges and found that the $x_{2}$ spectral range is the most useful because the region contains band heads for four of the five stable TiO isotopologues, which suffices to determine all titanium isotope abundances. The ${ }^{46} \mathrm{Ti} \mathrm{O}$ band head is severely blended by telluric feature, but ${ }^{46} \mathrm{Ti}$ can be determined as ${ }^{46} \mathrm{Ti}=100-\left({ }^{47} \mathrm{Ti}+{ }^{48} \mathrm{Ti}+{ }^{49} \mathrm{Ti}+{ }^{50} \mathrm{Ti}\right)$.

Our analysis of band heads in the $x_{2}$ spectral range provides more reliable abundances of $\mathrm{TiO}$ isotopologues, especially of ${ }^{50} \mathrm{TiO}$ and ${ }^{49} \mathrm{TiO}$, which are not blended by strong lines of ${ }^{48} \mathrm{TiO}$. Conversely, this spectral range is very interesting because some nucleosynthesis computations predict a large deviation of the ${ }^{50} \mathrm{Ti}$ and ${ }^{49} \mathrm{Ti}$ from the solar abundances at the early epochs of the evolution of our Universe, see the Introduction.

We developed a new procedure that allowed us to determine all $\mathrm{Ti}$ isotope abundances together with the Ti abundance using this spectral region. To determine the best solution, we applied a $5 \mathrm{D}$ minimisation procedure with input parameters ${ }^{47} \mathrm{Ti},{ }^{48} \mathrm{Ti}$, ${ }^{49} \mathrm{Ti},{ }^{50} \mathrm{Ti}$, and the titanium abundance [Ti] to take the dependence of the determined isotopic ratios on the adopted titanium abundance into account. In this way, we determined the titanium isotopic ratios and abundance of Ti from our analysis of $x_{2}$ spectral range in the framework of the self-consistent approach. This a the key part of our procedure that we developed to analyse $\mathrm{TiO}$ isotopologue abundances. Furthermore, to obtain statistically significant results, we implemented a special procedure as part of our solution determination: the sets of the best solutions were sorted in the ascending order of the minimisation sums $S$, and the corresponding isotopic ratios and $\mathrm{Ti}$ abundances were averaged across $S_{\min }-\Delta S \leq S \leq S_{\min }$, where $S_{\min }$ and $\Delta S$ are the minimum $S$ and the formal error of its determination $S$, see Sect. 2.4.

\subsection{Analysis of GJ $15 A$ and GJ $15 B$ spectra}

In our study, we modelled $\mathrm{TiO}$ spectra of two stars that form a binary system, specifically, the CARMENES spectra of two $\mathrm{M}$ dwarfs, GJ 15A and GJ 15B, in the spectral ranges of our interest. We selected GJ 15A and GJ 15B because (a) they are comparatively well studied and (b) they are slow rotators. We anticipated that the isotope abundance in both stars should be 
equal and thus we could use the binarity of the system to prove the reliability of our results when (a) we know all abundances in the atmospheres of both stars, (b) their effective temperatures are well determined from the fits to observed spectral energy distributions; (c) model atmospheres are computed for the found $T_{\text {eff }}, \log g$ and abundances, and d) spectra of both components were observed with the same high quality. Unfortunately, none of these conditions were entirely satisfied. We fixed $T_{\text {eff }}=3800 \mathrm{~K}$ for GJ $15 \mathrm{~A}$, and $T_{\text {eff }}=3500 \mathrm{~K}$ for GJ $15 \mathrm{~B}$, and adopted $\log \mathrm{g}=5.0$ for both stars because the system is rather old, see Table 1. Furthermore, GJ $15 \mathrm{~B}$ is fainter, and the $\mathrm{S} / \mathrm{N}$ of the obtained spectrum for the cooler dwarfs is lower than that of GJ 15A.

Our best fits to the observed spectra of GJ 15A and GJ 15B in the $x_{2}$ spectral range provide non-solar Ti isotopic ratios of ${ }^{46} \mathrm{Ti}$, ${ }^{47} \mathrm{Ti},{ }^{48} \mathrm{Ti},{ }^{49} \mathrm{Ti}$, and ${ }^{50} \mathrm{Ti}$ of $7.9,5.2,72.8,7.9$, and 6.2 for GJ $15 \mathrm{~A}$ (M1 V) and 7.4, 4.2, 76.6, 5.8, and 6.0 for GJ 15B (M3 V) with an accuracy of \pm 0.2 . Furthemore, [Ti] $=0.040$ and 0.199 with an accuracy \pm 0.10 was also determined for GJ $15 \mathrm{~A}$ and GJ $15 \mathrm{~B}$, respectively. The differences in the isotope abundances between the two stars are significantly larger than the uncertainties of each individual determination. It is unclear whether these differences reflect actual differences in the isotope abundances in the two stellar objects, inaccuracies in the determination of the relevant physical properties of both systems (e.g. temperature), or can be attributed to modelling uncertainties. For more definite conclusions, an abundance analysis for all elements should be considered in both stars. Ideally, the observed spectra of both $\mathrm{M}$ dwarfs should be of the same quality, that is, have the same resolution and $\mathrm{S} / \mathrm{N}$.

We note some disagreement in the shape and intensity between the computed molecular bands of ${ }^{50} \mathrm{Ti} \mathrm{O}$ and the observations. Likely, here we have some contribution of other unidentified molecule(s). Given the promise of this spectral region in determining titanium isotope abundances, we suggest detailed consideration of this issue in the future.

We anticipated that pecularities in abundances can be explained by the inaccuracies of $T_{\text {eff }}$ determination, for instance, we found that lowering $T_{\text {eff }}$ of GJ $15 \mathrm{~A}$ by $100 \mathrm{~K}$ reduces the $\mathrm{Ti}$ abundance for the star by -0.15 . Furthermore, the Ti I line seen in the lower panels of Fig. A.3 can be fitted by adopting $[\mathrm{Ti}]=-0.2$. However, this $\mathrm{Ti}$ abundance describes the $\mathrm{Ti}$ line in GJ 15A better. To describe the Ti line in the GJ 15B spectrum, we should adopt a larger titanium abundance. Furthermore, from the fit to the $x_{2}$ spectral range, we obtained larger [Ti] in the atmosphere of GJ 15B.

Of note, we found that the binary seems to be Fe deficient, as previously reported by several authors, see Table 1 . To obtain reasonable fits to the two Fe I lines observed in the $x_{2}$ spectral range, we adopted $[\mathrm{Fe}]=-0.4$, see Fig. 3. Conversely, we obtained a rather solar abundance of $\mathrm{Ti}$ in the atmospheres of two stars. Temperature changes cannot explain the observed overabundance of $\mathrm{Ti}$ with respect to $\mathrm{Fe}$. The overabundance of $\mathrm{Ti}$ with respect to Fe can be interpreted, at least qualitatively, as due to an $\alpha$-element abundance enhancement in metal-poor stars, see the Introduction.

\section{Conclusions}

An accurate determination of titanium isotope abundances can shed new light on astrophysical processes. On the large scale, comparing isotope abundances in atmospheres of the slowly evolving $\mathbf{M}$ dwarfs to abundances in more massive, fast evolving $M$ giants could provide new information about the evolutionary processes in our Galaxy. In individual astrophysical systems, such as the Earth-Moon system and host star-exoplanet systems, more local histories can be determined, for example, nearidentical titanium (and oxygen) isotope abundances on Earth and the Moon challenge the dominant theory that the Moon formed after a giant impact, see the Introduction.

Our results demonstrate that titanium isotope abundances in $M$ stars can be most effectively measured using high-resolution spectroscopy of $\mathrm{TiO}$ in the $x_{2}$ spectral region, that is, air wavelengths of 7580-7594 A, through fitting observed spectral intensities of many isotopologue features against models computed using the Тото line list. The successes and limitations of this method were explored through detailed studies of the spectroscopy of the GJ $15 \mathrm{~A}$ and GJ $15 \mathrm{~B}$ binary system. We find that for optimal results, $T_{\text {eff }}, \log g$, and the abundances of all elements should be determined by the most accurate procedure and the spectra of all $\mathrm{M}$ dwarfs should be of the same very high quality. Taking into account all uncertanties of our analysis, we conclude that our determinations are accurate to $\sim 5 \%$ of the absolute abundance of the main isotopologue and to $20-30 \%$ of the relative abundances of minor isotopologues. The use of better quality spectra will increase these accuracies by a factor of 10. Fortunately, the spectral range of our interest, that is, $x_{2}$, lies in the NIR, where observed fluxes are higherer than in optical wavelengths, and detectors provide data of higher $\mathrm{S} / \mathrm{N}$.

Acknowledgements. This study was funded as part of the routine financing programme for institutes of the National Academy of Sciences of Ukraine. Spectroscopic data calculated by the ExoMol group (funded by ERC as part of the Advanced Investigator 267219 project and UK Science and Technology Research Council (STFC) No. ST/R000476/1), the SIMBAD database (CDS, Strasbourg, France), and the Gaia spacecraft data (European Space Agency) were used. This study is based in part on archival data obtained using the infrared telescope operated by the University of Hawaii under a cooperative agreement with NASA. Authors would like to thank the SAO/NASA ADS team for the development and support of this remarkable data system. This work has made use of data from the European Space Agency (ESA) mission Gaia (https: //www. cosmos.esa.int/gaia), processed by the Gaia Data Processing and Analysis Consortium (DPAC, https://www.cosmos.esa.int/web/ gaia/dpac/consortium). Funding for the DPAC has been provided by national institutions, in particular the institutions participating in the Gaia Multilateral Agreement. We thank CARMENES team for providing high quality database of M-dwarf spectra which was used in this paper. We thank the anonymous referee for a thorough review and we highly appreciate the comments and suggestions, which significantly contributed to improving the quality of the paper.

\section{References}

Alexander, C. M. O., \& Nittler, L. R. 1999, ApJ, 519, 222

Allard, F. 2014, IAU Symp., 299, 271

Allard, F., Hauschildt, P. H., \& Schwenke, D. 2000, ApJ, 540, 1005

Anders, E., \& Grevesse, N. 1989, Geochim. Cosmochim. Acta, 53, 197

Bernath, P. 2020, ApJ, 895, 87

Cenarro, A. J., Peletier, R. F., Sánchez-Blázquez, P., et al. 2007, MNRAS, 374, 664

Chavez, J., \& Lambert, D. L. 2009, ApJ, 699, 1906

Ciddor, P. E. 1996, Appl. Opt., 35, 1566

Clegg, R. E. S., Lambert, D. L., \& Bell, R. A. 1979, ApJ, 234, 188

De Bievre, P., \& Taylor, P. 1993, Int. J. Mass Spectr. Zon Process., 123, 149

Furtenbacher, T., Császár, A. G., \& Tennyson, J. 2007, J. Mol. Spectr., 245, 115

Gaia Collaboration (Brown, A. G. A., et al.) 2018, A\&A, 616, A1

Gaidos, E., \& Mann, A. W. 2014, ApJ, 791, 54

Gaidos, E., Mann, A. W., Lépine, S., et al. 2014, MNRAS, 443, 2561

Gautier, T. N., I., Rieke, G. H., Stansberry, J., et al. 2007, ApJ, 667, 527

Hinkle, K., Wallace, L., \& Livingston, W. 1995, PASP, 107, 1042

Hoeijmakers, H. J., de Kok, R. J., Snellen, I. A. G., et al. 2015, A\&A, 575, A20

Howard, A., Marcy, G., Fischer, D., et al. 2014, ApJ, 794, 1

Hughes, G. L., Gibson, B. K., Carigi, L., et al. 2008, MNRAS, 390, 1710

Kobayashi, S., Nishimiya, N., \& Suzuki, M. 2019, Spectrochim. Acta Part B At. Spectr., 152, 30 
Koleva, M., \& Vazdekis, A. 2012, A\&A, 538, A143

Kurucz, R. L. 2011, Can. J. Phys., 89, 417

Lambert, D. L., \& Luck, R. E. 1977, ApJ, 211, 443

Lambert, D. L., Mallia, E. A., \& Smith, G. 1972, Sol. Phys., 26, 250

Lippincott, S. L. 1972, AJ, 77, 165

Lodders, K., Palme, H., \& Gail, H. P. 2009, Landolt Börnstein, 4B, 712

Mann, A. W., Feiden, G. A., Gaidos, E., Boyajian, T., \& von Braun, K. 2015, ApJ, 804, 64

McKemmish, L. K., Masseron, T., Sheppard, S., et al. 2017, ApJS, 228, 15

McKemmish, L. K., Masseron, T., Hoeijmakers, J., et al. 2019, MNRAS, 488, 2836

Mochizuki, Y., \& Kumagai, S. 2004, AIP Conf. Proc., 704, 369

Mochizuki, Y., Takahashi, K., Janka, H. T., Hillebrand t, W., \& Diehl, R. 1999, A\&A, 346, 831

Newton, E. R., Charbonneau, D., Irwin, J., et al. 2014, AJ, 147, 20

Passegger, V. M., Reiners, A., Jeffers, S. V., et al. 2018, A\&A, 615, A6

Pavlenko, Y. V. 1997, Astron. Rep., 41, 537

Pavlenko, Y. V. 2014, Astron. Rep., 58, 825

Pavlenko, Y. V., Rebolo, R., Martin, E. L., \& Garcia Lopez, R. J. 1995, A\&A, 303, 807

Pavlenko, Y., Suárez Mascareño, A., Rebolo, R., et al. 2017, A\&A, 606, A49

Pavlenko, Y. V., Yurchenko, S. N., \& Tennyson, J. 2020, A\&A, 633, A52

Piette, A. A., Madhusudhan, N., McKemmish, L. K., et al. 2020, MNRAS, 496, 3870

Plez, B. 1998, A\&A, 337, 495

Polyansky, O. L., Kyuberis, A. A., Lodi, L., et al. 2017, MNRAS, 466, 1363

Prugniel, P., Vauglin, I., \& Koleva, M. 2011, A\&A, 531, A165

Reid, I. N., Hawley, S. L., \& Gizis, J. E. 1995, AJ, 110, 1838
Reiners, A., Zechmeister, M., Caballero, J. A., et al. 2018, A\&A, 612, A49

Rudolf, N., Günther, H. M., Schneider, P. C., \& Schmitt, J. H. M. M. 2016, A\&A, 585, A113

Ryabchikova, T., \& Pakhomov, Y. 2015, Balt. Astron., 24, 453

Schlieder, J. E., Lépine, S., Rice, E., et al. 2012, AJ, 143, 114

Schwenke, D. W. 1998, Faraday Discuss., 109, 321

Taylor, G. J. 2012, Titanium Isotopes Provide Clues to Lunar Origin, Planetary Science Research Discoveries Report

Tennyson, J. 2019, Astronomical Spectroscopy : An Introduction to the Atomic and Molecular Physics of Astronomical Spectra, 3rd edn. (Singapore: World Scientific)

Tennyson, J., \& Yurchenko, S. N. 2012, MNRAS, 425, 21

Tennyson, J., Yurchenko, S. N., Al-Refaie, A. F., et al. 2016a, J. Mol. Spectr., 327,73

Tennyson, J., Lodi, L., McKemmish, L. K., \& Yurchenko, S. N. 2016b, J. Phys. B: At. Mol. Opt. Phys., 49, 102001

Terrien, R. C., Mahadevan, S., Deshpande, R., \& Bender, C. F. 2015, ApJS, 220, 16

Timmes, F. X., Woosley, S. E., \& Weaver, T. A. 1995, ApJS, 98, 617

Tóbiás, R., Furtenbacher, T., Tennyson, J., \& Császár, A. G. 2018, Phys. Chem. Chem. Phys., 21, 3473

Valenti, J. A., Piskunov, N., \& Johns-Krull, C. M. 1998, ApJ, 498, 851

van Buren, D., Brundage, M., Ressler, M., \& Terebey, S. 1998, AJ, 116, 1992

Woosley, S. E., \& Weaver, T. A. 1994, ApJ, 423, 371

Wyckoff, S., \& Wehinger, P. 1972, ApJ, 178, 481

Yurchenko, S. N., Lodi, L., Tennyson, J., \& Stolyarov, A. V. 2016, Comput. Phys. Commun., 202, 262

Zboril, M., \& Byrne, P. B. 1998, MNRAS, 299, 753

Zhang, J., Dauphas, N., Davis, A., Leya, I., \& Fedkin, A. 2012, Nat. Geosci., 5, 251 
Appendix A: Some additional stuff

Table A.1. Example of input data of ${ }^{47} \mathrm{Ti} \mathrm{O}$ lines in our computation.

\begin{tabular}{lcc}
\hline \hline$\lambda(\mathrm{A})$ in air & $g f$ & $E^{\prime \prime}(\mathrm{eV})$ \\
\hline 3493.707 & $3.172 \mathrm{E}-05$ & 0.890 \\
3493.707 & $3.172 \mathrm{E}-05$ & 0.890 \\
3494.907 & $3.323 \mathrm{E}-05$ & 0.897 \\
3494.907 & $3.323 \mathrm{E}-05$ & 0.897 \\
3496.134 & $3.481 \mathrm{E}-05$ & 0.903 \\
3496.134 & $3.481 \mathrm{E}-05$ & 0.903 \\
3496.479 & $3.521 \mathrm{E}-05$ & 0.917 \\
3496.479 & $3.521 \mathrm{E}-05$ & 0.917 \\
3497.389 & $3.647 \mathrm{E}-05$ & 0.910 \\
3497.389 & $3.647 \mathrm{E}-05$ & 0.910 \\
$\ldots$ & & \\
\hline
\end{tabular}
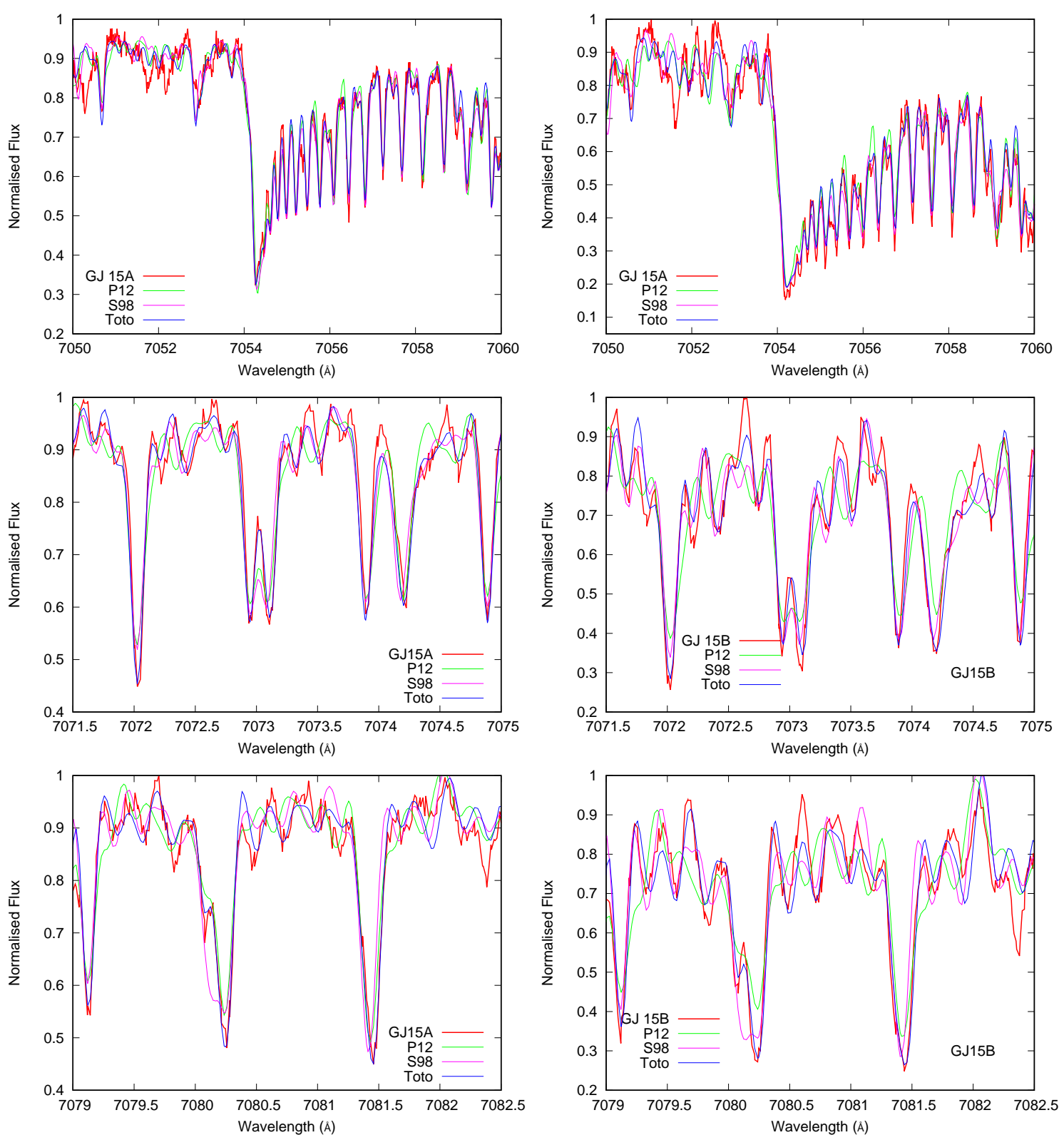

Fig. A.1. Comparison of fits to the observed TiO features in the $x_{1}$ spectral range in GJ 15A (left panels) and GJ 15B (right panels) using line lists of different authors. Here solar isotopic ratios of Ti were adopted. 
Ya. V. Pavlenko et. al.: Analysis of the TiO spectra
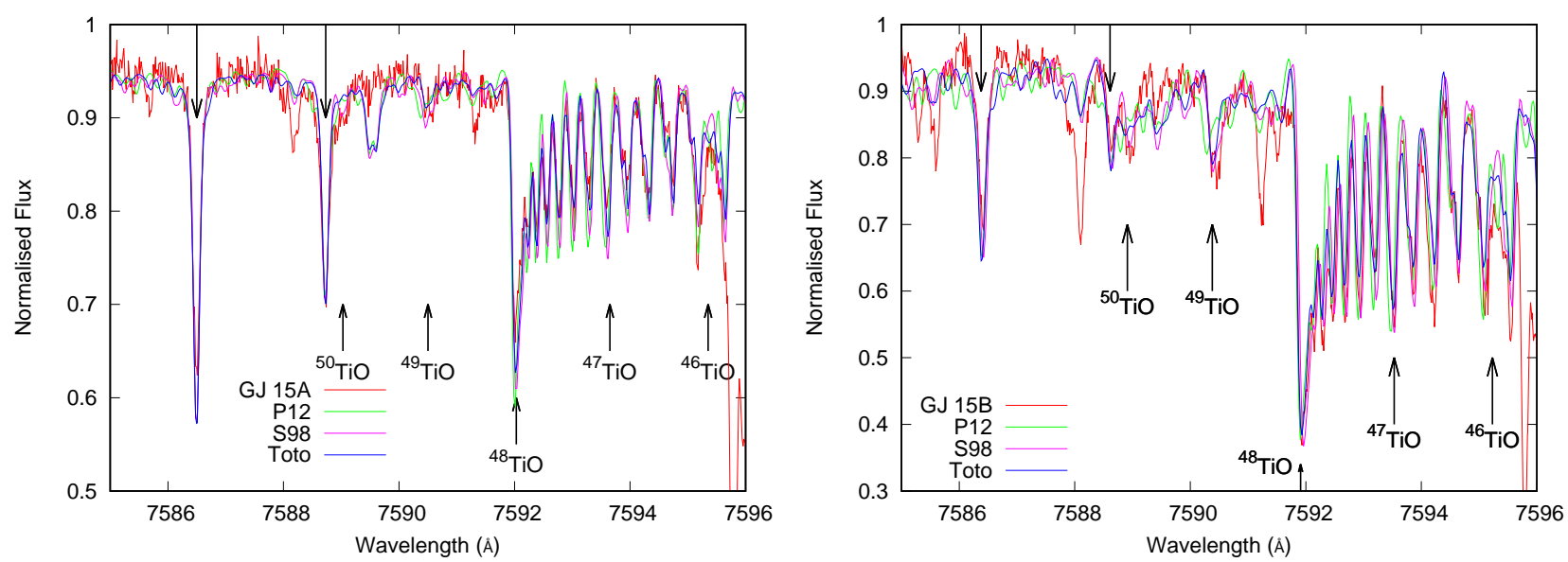

Fig. A.2. Comparison of fits to the observed TiO features in the $x_{2}$ spectral range in GJ 15A (left panel) and GJ 15B (right panel) using line lists of different authors. Here solar isotopic ratios of Ti were adopted.
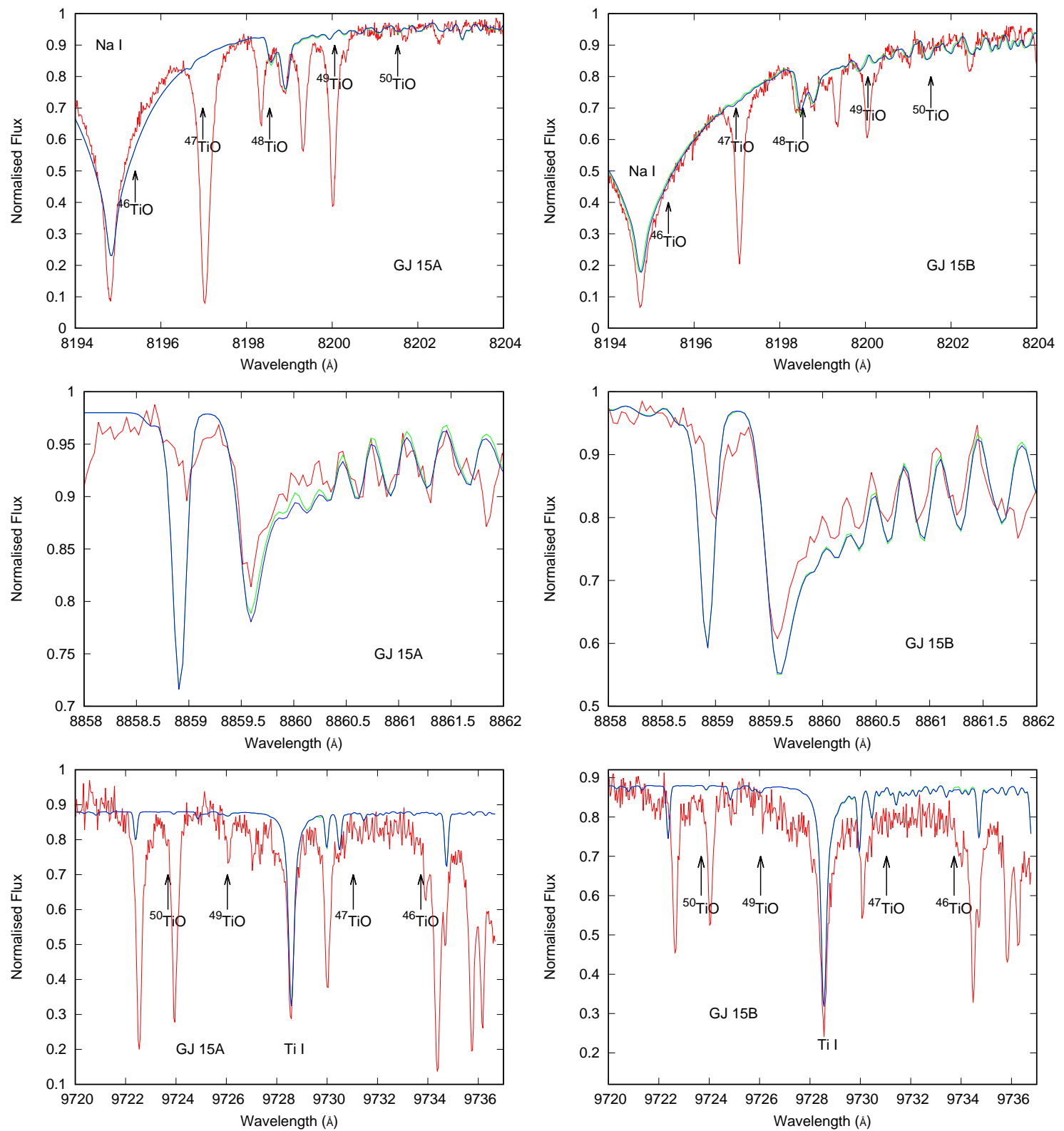

Fig. A.3. Top left: comparison of observed and computed TiO isotopic ratio spectra of GJ 15A and GJ 15B in the $x_{3}$ (top panels), $x_{4}$ (middle panels), and $x_{5}$ (bottom panels) spectral ranges for solar (green line) and M25 (blue line) abundance ratios. 